\title{
Inflation Scheme Derived from Universal Wave Function Interpretation of String Theory
}

\author{
Dr. Zhi Gang Sha ${ }^{1}$ and Rulin Xiu ${ }^{2}$ \\ 1. Institute of Soul Healing and Enlightenment, 30 Wertheim Court, Unit 27D, Richmond Hill, Ontario L4B 1B9, Canada \\ 2. Hawaii Theoretical Physics Research Center, 16-266 E. Kipimana St, Keaau, HI 96749, USA
}

\begin{abstract}
Inflation is the most commonly accepted theory in cosmology to explain why the universe appears flat, homogeneous, and isotropic, as well as the origin of the large-scale structure of the cosmos and why no magnetic monopoles have been detected. However, so far, the origin of the inflation epoch and what is the hypothetical field, the inflation, giving rise to inflation, remains unknown. String theory is one of the promising candidates for the grand unification theory. Grand unification theory is to use one mathematic formula to describe everything. In this work, we study the inflation scheme in a new development in string theory, UWFIST (universal wave function interpretation of string theory). We show that from UWFIST we can derive from the fundamental theory that the long-range vibration is the possible candidate of inflation. We estimate the vacuum energy created by the long-range vibration and show that it can indeed drive the inflation.
\end{abstract}

Key words: Inflation, string theory, universal wave function interpretation of string theory, grand unification theory.

\section{Introduction}

In physical cosmology, inflation is a theory of exponential expansion of space in the early universe. Inflation theory proposes a rapid exponential expansion of the early universe by a factor of at least $10^{78}$ in volume, driven by a negative-pressure vacuum energy density $[1,2]$. The inflationary epoch lasted from $10^{-36}$ seconds to $10^{-33}$ and $10^{-32}$ seconds after the big bang. Following the inflationary period, the universe continues to expand, but at a less rapid rate $[1,2]$.

Developed in the early 1980s, inflation is so far the mostly accepted way to solve the fine-tuning problems with the initial conditions of the universe. Inflation hypothesis can show why our universe appears flat, homogeneous, and isotropic. It can account for the origin of the large-scale structure of the cosmos and why no magnetic monopoles have been detected.

The hypothetical particle or field thought to be responsible for inflation is called inflation. However,

Corresponding author: Rulin Xiu, Ph.D., research field: theoretical physics. the detailed particle physics mechanism responsible for inflation or inflation remains unknown. Furthermore, the abundance of "bad inflation" in the quantum theory and the realization that inflation is eternal has been challenging the real predictive power of inflation theory [3-6].

In this work we will derive the possible inflation and the energy created by the inflation for the inflation from a new development in string theory, the UWFIST (universal wave function interpretation of string theory).

String theory is a promising candidate for the grand unification theory $[7,8]$. How to understand and derive testable results from string theory has been a long debated subject. The currently accepted interpretation is that string theory studies the quantum dynamics of a string. The vibration of string creates particles and interactions. The scale of the string is set to be Planck Scale (approximately $1.6 \times 10^{-35}$ meters). String theory has the potential to predict the dimension of space-time, the particle and interaction spectrum, to unify all the forces and fundamental particles, and more. However, with all these great 
potentials and promises, current string theory has not yet made many testable predictions.

In our previous work $[9,10]$ by combining quantum physics and general relativity, we derive a space-time uncertainty relationship, which suggests that the wave function of universe and everything is created from a string like action. We call this the UWFIST. UWFIST indicates the existence of long-range quantum vibrations in our universe. These long-range vibrations could be the source of dark energy. From the wave function of universe, we can calculate or estimate the vacuum energy of our universe. In Ref. [9] we have demonstrated that using the age of our universe and fundamental constants such as the gravitational constant $\mathrm{G}$, Planck constant $\hbar$, and speed of light, UWFIST may yield the estimate of vacuum energy consistent with the current experimental observation. We find Ref. [10] that these large-scale quantum vibrations can cause and explain the observed large-scale anisotropies, anomalous alignments, and non-Gaussian distributions in the cosmic microwave background radiation as well as in other background matter and radiation.

In this paper, we will briefly review the derivation of the UWFIST. We will show that there exist the long-range vibrations in UWFIST. We will show that these long-range vibrational fields can generate the vacuum energy density, which can drive the inflation in the early age of the universe. They are the possible candidates for inflation.

\section{A New Way to Derive and Interpret String Theory}

In our previous work, we show that when we take both the effect of quantum physics and gravity into consideration, we obtain an uncertainty relation between space measurement $\Delta \sigma$ and time measurement $\Delta \tau$ in causal region:

$$
\Delta \sigma \Delta \tau \geq 1_{\mathrm{p}} \mathrm{t}_{\mathrm{p}}
$$

Here $l_{p}$ is the Planck length, $l_{p}=\left(\hbar G / c^{3}\right)^{1 / 2}=1.616$ $\times 10^{-35}$ meter. And $t_{p}$ is the Planck time, $t_{p}=\left(\hbar G / c^{5}\right)^{1 / 2}$
$=5.39 \times 10^{-44}$ second.

We suggest that this relation indicates that space and time are quantized and the non-commutation relation between space and time:

$$
[\sigma, \tau]=\mathrm{il}_{\mathrm{p}} \mathrm{t}_{\mathrm{p}}
$$

Using the quantization procedure in quantum physics [11], we propose that the space time uncertainty relation (1) and non-commutation relation (2) indicates a string action $A_{s}$ in the form:

$$
\mathrm{A}_{\mathrm{s}}=\int \mathrm{d} \tau \mathrm{d} \sigma / \mathrm{l}_{\mathrm{p}} \mathrm{t}_{\mathrm{p}}
$$

Action $\mathrm{A}_{\mathrm{s}}$ evolves the wave function from $\psi(0,0)$ at the space 0 and time 0 to $\psi(\mathrm{L}, \mathrm{T})$ at the space $\mathrm{L}$ and time $\mathrm{T}$ in the following way:

$$
\begin{gathered}
\psi(\mathrm{L}, \mathrm{T})=\Sigma_{\text {sum over all possible paths }} \exp \left(\mathrm{i} \int_{0}{ }_{\mathrm{T}} \mathrm{d} \tau \int_{0}{ }_{\mathrm{L}} \mathrm{d} \sigma / \mathrm{l}_{\mathrm{p}} \mathrm{t}_{\mathrm{p}}\right) \\
\psi(0,0)
\end{gathered}
$$

The Eq. (4) is the UWFIST.

UWFIST deviates from the usual interpretation of string theory in two ways. Firstly, the world-sheet space $\sigma$ and time $\tau$ integration of the Eq. (3) is over the causal region i.e.

$$
\mathrm{A}_{\mathrm{s}}=\int_{0}{ }^{\mathrm{T}} \mathrm{d} \tau \int_{0}{ }^{\mathrm{L}} \mathrm{d} \sigma / \mathrm{l}_{\mathrm{p}}{ }^{2}
$$

Here $\mathrm{T}$ and $\mathrm{L}$ are the age and length of the causal horizon. They relate to the age and length of the horizon of our universe. In the normal interpretation of string theory, $\sigma$ is the string coordinate. $\mathrm{L}$ is taken to be the length of the string. It is set to be Planck length $1_{\mathrm{p}}$. In Eq. (4), $\mathrm{T}$ and $\mathrm{L}$ are variables. They change as universe evolves or as space and time pass by. The second difference between the usual string theory and UWFIST is that the universal wave function $\Psi(\mathrm{L}, \mathrm{T})$ are introduced. Here $\Psi(0,0)$ and $\Psi(\mathrm{L}$, $\mathrm{T})$ represents the universal wave function at the initial space and time and at the space $\mathrm{L}$ and time $\mathrm{T}$. We propose that the universal wave function could be the wave function of our universe. This is because if we extend the string action to the cases of superstring and heterotic string with background fields, the universal wave function $\Psi$ can include all fundamental particles, all gauge interactions, gravity, and more. It also includes the large structures of our universe up to the scale of horizon. In other words, UWFIST could be 
the wave function of our universe. Except for these two major conceptual differences, the usual string theory calculation can still be applied in UWFIST.

In the string theory, there are two sets of space-time: the 2-dimensional world-sheet $(\sigma, \tau)$ and the observed space-time $X^{\mu}(\sigma, \tau)$. The observed space-time $X^{\mu}(\sigma, \tau)$ is a projection from the world sheet to possibly a higher dimensional space-time. In terms of $\mathrm{X}^{\mu}(\sigma, \tau)$, the action $A_{s}$ in Eq. (3) becomes:

$$
A_{s}=\left(1 / l_{p} t_{p}\right) \int d \tau d \sigma g^{1 / 2} g^{a b} \partial a X \mu \partial b X^{\mu}
$$

Here $\mathrm{g}^{\alpha \beta}$ is the metric tensor on the world-sheet and $\mathrm{g}=-\operatorname{det} \mathrm{g}^{\alpha \beta}$. As shown in string theory $[7,8]$, the general form of Eq. (5) in the presence of massless background field is in the form:

$$
\begin{gathered}
A_{s}=\left[i \alpha \int _ { 0 } ^ { T } d \tau \int _ { 0 } ^ { L } d \sigma g ^ { 1 / 2 } \left(g^{\alpha \beta} G^{\mu v} \partial_{\alpha} X_{\mu} \partial_{\beta} X_{v}+\right.\right. \\
\left.\left.\varepsilon^{\alpha \beta} B^{\mu v} \partial_{\alpha} X_{\mu} \partial_{\beta} X_{v}+1 / 4 \alpha \Phi R\right)\right]
\end{gathered}
$$

Here $\mathrm{G}^{\mu v}(\tau, \sigma), \mathrm{B}^{\mu v}(\tau, \sigma), \Phi(\tau, \sigma)$ are metric tensor, anti-symmetric tensor, and scalar background fields on the observed space. And $\mathrm{R}$ is the scalar curvature related to $\mathrm{g}^{\alpha \beta}$. The universal wave function in the presence of background fields is:

$$
\begin{gathered}
\Psi\left(X^{\mu}(L, T), G^{\mu v}(L, T), B^{\mu v}(L, T), \Phi(L, T)\right)= \\
\int D X^{\mu} D g^{\mu v} D B^{\mu v} D \Phi \exp \left[i \alpha \int_{0}^{T} d \tau \int_{0}^{L} d \sigma\right. \\
\left.g^{1 / 2}\left(g^{\alpha \beta} G^{\mu v} \partial_{\alpha} X_{\mu} \partial_{\beta} X_{v}+\varepsilon^{\alpha \beta} B^{\mu v} \partial_{\alpha} X_{\mu} \partial_{\beta} X_{v}+1 / 4 \alpha \Phi R\right)\right](7)
\end{gathered}
$$

We can extend $\Psi\left(X^{\mu}(L, T), G^{\mu v}(L, T), B^{\mu v}(L, T)\right.$, $\Phi(\mathrm{L}, \mathrm{T}))$ to include other massive vibrations. We can also extend the above string action to the cases of superstring and heterotic string to include all fundamental particles, all gauge interactions, gravity, and more. This universal wave function tells us the probability for our universe to be at space-time coordinate $X^{\mu}(L, T)$ and background fields $G^{\mu v}(L, T)$, $\mathrm{B}^{\mu \nu}(\mathrm{L}, \mathrm{T}), \quad \Phi(\mathrm{L}, \mathrm{T})$. We can do a Fourier transformation to transform the wave function $\Psi\left(\mathrm{X}^{\mu}(\mathrm{L}\right.$, $\left.\mathrm{T}), \mathrm{G}^{\mu v}(\mathrm{~L}, \mathrm{~T}), \mathrm{B}^{\mu v}(\mathrm{~L}, \mathrm{~T}), \Phi(\mathrm{L}, \mathrm{T})\right)$ to be expressed in the vibrational space expressed by the various vibrations $\left(\omega^{\mu}, \kappa^{\mu v}, \ldots\right)$. The wave function $\Psi\left(\omega^{\mu}(L, T)\right.$, $\left.\kappa^{\mu v}(L, T), \ldots\right)$ expresses what kind of vibrations in our universe and how much they exist at proper time $\mathrm{T}$ and horizon length $\mathrm{L}$ in our universe.

\section{Inflation and the Energy Driving Inflation}

A natural indication and consequence of UWFIST is the existence of long-range vibrations. The space-time coordinate $\mathrm{X}^{\mu}(\tau, \sigma)$ is composed of vibrations in the form $\exp [i \pi \mathrm{n}(\sigma+\mathrm{c} \tau) / \mathrm{L}]$ and $\exp [i \pi n(\sigma-c \tau) / L]$. Here $n$ is an integer. Unlike the normal string theory, in which $\mathrm{L}$ is the Planck length, now $\mathrm{L}$ is causal horizon distance. The largest wavelength is on the order of the length of the causal horizon. These vibrations are very fine. They are very dark in the sense that it is very difficult to detect them. In fact, to detect the vibration with the wavelength $\mathrm{L}$, it takes the time $\mathrm{L} / \mathrm{c}$. If $\mathrm{L}$ is the horizon distance, this means that it takes the time of the age of the visible universe or a detector as large as the whole visible universe to detect these vibrations.

We propose that these long-range vibrations can be the possible candidates of inflation. To demonstrate this, we calculate the vacuum energy density created by them. Here we define the vacuum energy to be the lowest energy state of the universal wave function.

Because our observed universe is the projection from the world-sheet, the world sheet contains all the information, energy, and matter about our universe. All the vibrations in the observed space-time come from the vibrations in the world-sheet. Therefore, we simply need to calculate the vacuum energy in the two-dimensional world sheet.

To calculate the vacuum energy of the world-sheet, let's assume that on the world-sheet, the energy is in the form of harmonic oscillator. The energy at the state $(n, m)$ with the frequency $v_{n}=n / T$ and wavelength $\lambda_{\mathrm{m}}=\mathrm{L} / \mathrm{m}$ is:

$\mathrm{E}_{\mathrm{n}, \mathrm{m}}=(\mathrm{n}+1 / 2) \mathrm{h} / \mathrm{T}$.

The lowest energy of this vacuum fluctuation is $\mathrm{E}_{0 \mathrm{n}, \mathrm{m}}=\mathrm{h} / 2 \mathrm{~T}$.

The number of possible vibrations on the world sheet is $\mathrm{TL} /\left(\mathrm{t}_{\mathrm{p}} \mathrm{l}_{\mathrm{p}}\right)$. We can get this from the uncertainty relation in Eq. (1). To make the space measurement $\Delta \sigma$, we should at least use the vibration with the wavelength $\lambda_{\mathrm{m}}=\mathrm{L} / \mathrm{m} \sim \Delta \sigma$. To measure the time 
measurement $\Delta \tau$, we should at least use the vibration with the frequency $v_{\mathrm{n}}=\mathrm{n} / \mathrm{T} \sim \Delta \tau$. Since $\Delta \sigma \Delta \tau \geq \mathrm{l}_{\mathrm{p}} \mathrm{t}_{\mathrm{p}}$, we get $\mathrm{nm}<\mathrm{TL} /\left(\mathrm{t}_{\mathrm{p}} \mathrm{l}_{\mathrm{p}}\right)$.

The total lowest vacuum energy is:

$$
\mathrm{E}_{\mathrm{vac}}=\mathrm{h} / 2 \mathrm{~T} \times \mathrm{cTL} / \mathrm{l}_{\mathrm{p}}^{2}=\mathrm{hLc} /\left(2 \mathrm{l}_{\mathrm{p}}^{2}\right)
$$

It is proportional to the length of the causal horizon. The lowest vacuum energy density in the three dimensional space is:

$$
\rho_{\mathrm{vac}}=\mathrm{E}_{\mathrm{vad}} /\left(4 \pi \mathrm{L}^{3} / 3\right)=\rho_{\mathrm{p}} \mathrm{l}_{\mathrm{p}}^{2} /\left(2 \mathrm{~L}^{2}\right)
$$

Here $\rho_{\mathrm{p}}$ is the Planck energy density,

$$
\rho_{\mathrm{p}}=\mathrm{E}_{\mathrm{p}} /\left(4 \pi \mathrm{l}_{\mathrm{p}}^{3} / 3\right)
$$

and $E_{p}$ is the Planck energy $E_{p}=\hbar / t_{p}=1.956 \times 10^{9}$ Joul, $t_{p}$ and $l_{p}$ are the Planck time and Planck length $l_{p}$ $=\mathrm{ct}_{\mathrm{p}}=\left(\hbar \mathrm{G} / \mathrm{c}^{3}\right)^{1 / 2}$.

The Eq. (9) tells us that the vacuum energy density observed in three space dimensions is inversely proportional to the square of the length of horizon $\mathrm{L}$. At the beginning stage of universe, the vacuum energy density is very large. For example, when our universe is of the size of the Planck scale $1_{p}$, the vacuum energy density is half of the Planck energy density $\rho_{\mathrm{vac}}=\rho_{\mathrm{p}} / 2$. This large vacuum energy density provides the energy source for inflation, the rapid initial expansion. Therefore, the result of Eq. (9) indicates the vacuum energy can be the energy source for inflation. As the universe expands to larger scale, the vacuum energy decreases, but is non-zero. Our universe will continue its accelerated expansion at much slower rate.

Now let us assume that $\mathrm{L}$ is the Hubble radius with $\mathrm{L}=\mathrm{c} / \mathrm{H}$. Here $\mathrm{c}$ is the speed of light and $\mathrm{H}$ is the Hubble constant. Hubble constant is the unit of measurement used to describe the expansion of the universe. It has also been used to estimate the age of our universe. With this assumption, we get:

$$
\begin{gathered}
\rho_{\text {vac }}=\mathrm{E}_{\mathrm{vac}} /\left(4 \pi \mathrm{L}^{3} / 3\right)=3 \mathrm{~h} /\left(8 \pi \mathrm{cL}^{2} 1_{\mathrm{p}}{ }^{2}\right)=3 \mathrm{c}^{2} /\left(8 \pi \mathrm{GL}^{2}\right)= \\
3 \mathrm{H}_{0}{ }^{2} /(8 \pi \mathrm{G})=\rho_{\mathrm{c}}
\end{gathered}
$$

Since the relation (8) is general for all $\mathrm{L}$ and $\mathrm{T}$, it shows that the lowest vacuum energy density is equal to the critical energy density for 4-dimensional space-time and remains to be so as universe expands. This may provide further theoretical explanation why our universe is flat, with its energy density is so close to critical energy density.

\section{Discussion and Conclusion}

In this paper, we propose a possible scheme of inflation derived from the UWFIST. We demonstrate that this new development of string theory indicates the existence of long-range vibrations. These long-range vibrations could be the inflation. We estimate the vacuum energy created by them and find that they can propel the exponential expansion, the inflation, at the early stage of the universe. At the later time, the vacuum energy generated by them decreases significantly and the acceleration of the expansion gets much less.

We conclude that UWFIST provides a simple explanation and calculable prediction from the first principle about why our universe experiences fast inflation at the early stage, continue to expand, and at the same time remains very flat. It predicts that the inflation is the long-range vibrations. UWFIST gives a new possible scheme for inflation.

In our previous work, we show that the vacuum energy created by the inflation, the long-range vibrations, propels the current accelerated expansion of the universe. They are the dark energy. Our calculation agrees with the current observation about dark energy [9]. It can also explain the large-scale correlation observed in the large-scale anisotropies and non-Gaussian distributions of the cosmic microwave background and other background field as well as the anomalous alignments of the quadrupole and octupole modes [10].

We suggest UWFIST, as the new development in string theory, has shown the promise to yield testable theoretical predictions in astrophysics and cosmology. It is worth further investigation.

\section{References}

[1] Guth, Alan. 1998. The Inflationary Universe. Basic Books.

[2] Steinhardt, Paul J., and Neil Turok. 2007. Endless 
Universe: Beyond the Big Bang. Doubleday.

[3] Penrose, Roger. 2004. The Road to Reality: A Complete Guide to the Laws of the Universe. London: Vintage Books, 755.

[4] Penrose, Roger. 1989. "Difficulties with Inflationary Cosmology". Annals of the New York Academy of Sciences 271: 249-64. Bibcode: 1989NYASA.571. 249P. doi: 10.1111/j.1749-6632.1989.tb50513.

[5] Dicke, R. H. 1961. "Dirac's Cosmology and Mach's Principle”. Nature $192 \quad$ (4801): 440-1. Bibcode1961Natur.192.440D. doi: 10.1038/192440a0.

[6] Steinhardt, Paul J. 2011. "The Inflation Debate: Is the Theory at the Heart of Modern Cosmology Deeply Flawed?" (Scientific American, April; pp. 18-25).

[7] Michael Green, John H. Schwarz and Edward Witten 1987. Superstring Theory. Cambridge University Press. Vol. 1: Introduction. ISBN 0-521-35752-7. Vol. 2: Loop
Amplitudes, Anomalies and Phenomenology. ISBN 0-521-35753-5.

[8] Polchinski, Joseph. 1998. String Theory. Cambridge University Press. Vol. 1: An Introduction to the Bosonic String. ISBN 0-521-63303-6. Vol. 2: Superstring Theory and Beyond. ISBN 0-521-63304-4.

[9] Sha, Zhigang, and Xiu, Rulin. 2017. "Dark Energy and Estimate of Cosmological Constant from String Theory." Journal of Astrophysics \& Aerospace Technology, accepted on Mar 27, 2017

[10] Sha, Zhigang, and Xiu, Rulin 2017. "Derivation of Large-Scale Anisotropy and Anomalous Alignment From Universal Wave Function Interpretation of String Theory." Submitted to Journal of Astrophysics \& Aerospace Technology.

[11] Feynman, Richard P., and Hibbs, Albert. 1965. Quantum Mechanics and Path Integrals. McGraw Hill. ISBN 0-07-020650-3. 